\title{
Segurança e eficácia dos stents farmacológicos eluidores de biolimus com polímero biodegradável: análise do registro EINSTEIN (Evaluation of Next-generation drug-eluting STEnt IN patients with coronary artery disease)
}

\author{
Safety and efficacy of biolimus-eluting stent with biodegradable polymer: \\ insights from EINSTEIN (Evaluation of Next-generation drug-eluting \\ STEnt IN patients with coronary artery disease) Registry
}

Cristiano Freitas de Souza ${ }^{1}$, Anwar Mohamed El Mouallem ${ }^{1}$, Fábio Sândoli de Brito Júnior ${ }^{1}$, Alexandre Antônio Cunha Abizaid ${ }^{1}$, Breno Oliveira Almeida ${ }^{1}$, Amanda Gonçalves Almeida ${ }^{1}$,

Teresa Cristina Dias Cunha Nascimento ${ }^{1}$, Marco Antonio Perin ${ }^{1}$, Adriano Caixeta ${ }^{1}$

\section{RESUMO}

Objetivo: Avaliar a incidência de eventos adversos cardíacos maiores (morte cardíaca, infarto agudo do miocárdio ou necessidade de nova revascularização do vaso-alvo) em 1 ano em pacientes do "mundo real". Métodos: 0 registro EINSTEIN é um estudo observacional prospectivo, unicêntrico, que incluiu consecutivamente 103 pacientes (152 lesões) tratados com o stent Biomatrix ${ }^{T M}$, eluidor de biolimus A9 e polímero biodegradável. Resultados: A média das idades foi de $65,0 \pm 12,4$ anos; 0 gênero masculino representou $83,5 \%$ dos pacientes; e 37,9\% deles eram diabéticos. Em 1 ano, eventos adversos cardíacos maiores ocorreram em $11,7 \%$ dos pacientes, incluindo $2,9 \%$ de morte cardíaca, $4,9 \%$ de infarto agudo do miocárdio sem supradesnivelamento do segmento ST e 3,9\% de revascularização do vaso-alvo. Trombose de stent esteve presente em apenas $1 \%$ (1 paciente) após o seguimento clínico de 1 ano. Conclusão: 0 presente registro sugere que os novos stents farmacológicos eluidores de biolimus A9 com polímero biodegradável são seguros e eficazes em pacientes da prática clínica diária, com baixas taxas de eventos adversos cardíacos maiores no longo prazo.

Descritores: Materiais biocompatíveis; Stents farmacológicos; Sistemas de liberação de medicamentos; Polímeros/sínstese química; Sirolimo/ análogos \& derivados; Doença da artéria coronariana/terapia

\section{ABSTRACT}

Objective: To evaluate the incidence of major adverse cardiac events (cardiac death, or acute myocardial infarct, or target vessel revascularization) at one year in "real world" patients. Methods: The EINSTEIN registry is an observational, prospective, single center study that consecutively included 103 patients (152 lesions) treated with the Biomatrix ${ }^{\mathrm{TM}}$ stent, a biolimus A9-eluting stent with biodegradable polymer. Results: The mean age was $65.0 \pm 12.4$ years; male gender represented $83.5 \%$ of the patients; and $37.9 \%$ of them were diabetic. At one-year, major adverse cardiac events occurred in $11.7 \%$ of the patients, including $2.9 \%$ of cardiac death, $4.9 \%$ of with non ST- segment elevation acute myocardial infarction, and $3.9 \%$ of target vessel revascularization. Stent thrombosis occurred in only $1 \%$ (1 patient) at one-yearfollow-up. Conclusion: The present Registry suggests that new generation biolimus $A 9$ drug-eluting stents are safe and effective in a "real world", all-comers patients, showing low rates of major cardiac adverse events on long-term follow-up.

Keywords: Biocompatible events; Pharmacological stents; Drugdelivery systems; Polymers/chemical synthesis; Sirolimus/analogs \& derivatives; Coronary artery disease/therapy

\section{INTRODUÇÃo}

Desde sua incorporação na prática médica, há uma década, os stents farmacológicos (SF) tiveram seu uso difundido em diversos cenários clínicos e anatômicos, devido à redução consistente nas taxas de reestenose. Comparados aos stents não farmacológicos, os SF de

Trabalho realizado no Hospital Israelita Albert Einstein, São Paulo, SP, Brasil.

1 Hospital Israelita Albert Einstein, São Paulo, SP, Brasil.

Autor correspondente: Adriano Caixeta - Hospital Israelita Albert Einstein, Cardiologia Intervencionista - Avenida Albert Einstein, $627 / 701$ - Morumbi - CEP: 05652-900 - São Paulo, SP, Brasil Tel.: (11) 2151-0434 - E-mail: adriano.caixeta@einstein.br

Data de submissão: 18/1/2013 - Data de aceite: 13/8/2013

Conflito de interesse: não há. 
primeira geração foram eficazes em reduzir a hiperplasia neointimal com consequente redução da perda luminal tardia e reestenose binária ${ }^{(1-3)}$. Subsequentemente, entretanto, pôde-se constatar um aumento nos índices de trombose tardia e muito tardia ${ }^{(4-6)}$. Apesar do mecanismo dos eventos trombóticos ainda não estarem totalmente elucidados, a reação de hipersensibilidade secundária à presença permanente de um polímero é uma das potencias variáveis nessa complexa equação $^{(7,8)}$. Nesse contexto, surgiram os SF de nova geração, como o Biomatrix ${ }^{\mathrm{TM}}$ (Biosensors International, Morges, Suisse). Uma de suas principais características é a presença de um polímero biodegradável composto por ácido polilático. Esse polímero é aplicado unicamente na face abluminal do stent, após a total liberação do fármaco, e metabolizado nas substâncias inócuas ácido carbônico e água. O Biomatrix ${ }^{\mathrm{TM}}$ carreia o fármaco biolimus A9, que é um composto imunossupressor e antiproliferativo da mesma família do sirolimus, porém com solubilidade dez vezes maior quando comparado a este. No estudo randomizado LEADERS, o stent Biomatrix $^{\mathrm{TM}}$ mostrou ser mais seguro que o SF de primeira geração Cypher ${ }^{\mathrm{TM}(9)}$. No entanto, resultados clínicos de longo prazo utilizando stent Biomatrix ${ }^{\mathrm{TM}}$ em pacientes do "mundo real" são escassos.

\section{OBJETIVO}

O objetivo primário do registro EINSTEIN (Evaluation of Next-generation drug-eluting STEnt IN patients with coronary artery disease) é avaliar a incidência de eventos adversos cardíacos maiores definidos como a ocorrência combinada de morte de origem cardíaca, infarto agudo do miocárdio ou necessidade de revascularização do vaso-alvo relacionados ao stent Biomatrix $^{\mathrm{TM}}$ aos 30 dias, 6 meses, 12 meses e anualmente até 3 anos de acompanhamento. Os objetivos secundários consistiram de: (1) sucesso do procedimento, definido como o sucesso técnico com estenose residual $\leq 30 \%$; (2) ocorrência de eventos adversos cardíacos maiores na fase intra-hospitalar; (3) eventos individuais de óbito, infarto agudo do miocárdio, necessidade de revascularização do vaso-alvo e trombose de stent aos 30 dias, 6 meses e 12 meses.

\section{MÉTODOS}

\section{Casuística e desenho do estudo}

$\mathrm{O}$ presente estudo consistiu no registro prospectivo e unicêntrico de pacientes consecutivos e não selecionados, avaliando o padrão de uso atual e os resultados clínicos de 12 meses com o stent eluidor de biolimus A9 Biomatrix $^{\mathrm{TM}}$. Durante o período de outubro de 2008 a dezembro de 2010, 103 pacientes com 152 lesões coro- nárias foram tratados com o stent Biomatrix $^{\mathrm{TM}}$ no setor de Cardiologia Intervencionista do Hospital Israelita Albert Einstein (HIAE), em São Paulo. Destes, 101 $(98,0 \%)$ completaram 1 ano de seguimento, representando o objeto de análise deste manuscrito. Com o intuito de representar a prática clínica diária, os critérios de inclusão foram permissivos, incluindo todos os pacientes submetidos à angioplastia de rotina ou de emergência com idade $>18$ anos. Por tratar-se de análise observacional e retrospectiva de banco de dados, o comitê de ética dispensou a necessidade de assinatura do termo de consentimento. O protocolo do registro foi aprovado pelo Comitê de Ética do HIAE n ${ }^{-}$11/1530 (CAAE: 0038.0.028.000-11).

\section{Procedimento de intervenção coronária e protocolo de antiagregação}

O protocolo antiplaquetário consistiu na utilização de ácido acetilsalicílico (AAS) e clopidogrel ou prasugrel previamente ao implante do stent. Após o procedimento, a recomendação era manter o AAS na dose de $100 \mathrm{mg} /$ dia por tempo indefinido e o clopidogrel na dose de $75 \mathrm{mg} /$ dia ou prasugrel na dose de $10 \mathrm{mg} / \mathrm{dia}$, por um período mínimo de 12 meses, seguindo a prática clínica corrente. Em relação ao procedimento terapêutico, a técnica e o número de stents implantados ficava a cargo do operador responsável.

\section{Definições}

As seguintes definições foram aplicadas aos eventos cardíacos referidos acima. Morte cardíaca foi considerada qualquer morte que não pudesse ser atribuída à causa não cardíaca. Infarto do miocárdio foi considerado quando ao menos um dos seguintes critérios esteve presente: elevação de CPK-MB superior a três vezes o limite superior da normalidade; eletrocardiograma com evidência de nova onda-Q patológica (durante 0,04 segundos ou mais) em pelo menos duas leituras contíguas com CPK-MB positiva. Reintervenção do vaso-alvo foi definida como qualquer tentativa ou realização com sucesso de reintervenção percutânea ou cirúrgica do vaso-alvo. As definições de trombose de stent seguiram aquelas propostas pelo Academic Research Consortium (ARC), sendo classificada em definitiva (trombo detectado e angiograficamente confirmado em qualquer stent colocado no vaso-alvo ou quando a confirmação patológica de trombose aguda em paciente com síndrome coronária aguda for feita), provável (trombose de stent definida como qualquer morte inexplicável dentro de 30 dias, ou como infarto do miocárdio do vaso-alvo sem confirmação angiográfica de trombose, ou outra lesão 
culpada identificável) e possível (trombose de stent definida como morte inexplicável após 30 dias $)^{(10)}$.

O seguimento clínico foi realizado por meio de entrevista por telefone ou visita médica aos 30 dias, 6 meses, 12 meses e então anualmente após o procedimento de implante do stent Biomatrix $^{\mathrm{TM}}$.

\section{Coleta e gerenciamento dos dados}

A elaboração e a implementação do registro ficaram sob responsabilidade do setor de Intervenção Cardiovascular do HIAE.

Tabela 1. Características clínicas, fatores de risco, apresentacao clinica e medicações adjuvantes

\begin{tabular}{|c|c|}
\hline & $n=103$ \\
\hline Idade, anos & $65,07 \pm 12,49$ \\
\hline Gênero masculino, \% (n/total) & $83,5(86 / 103)$ \\
\hline Índice de massa corporal, kg/m² & $27,54 \pm 4,17$ \\
\hline Hipertensão, \% (n/total) & $75,7(78 / 103)$ \\
\hline Hipercolesterolemia, \% (n/total) & $70,9(73 / 103)$ \\
\hline Tabagista, \% (n/total) & $25,2(26 / 103)$ \\
\hline Diabetes mellitus, \% (n/total) & $37,9(39 / 103)$ \\
\hline Insulino-dependente & $5,8 \%(6 / 103)$ \\
\hline IAM prévio, \% (n/total) & $12,6(13 / 103)$ \\
\hline ATC prévia, \% (n/total) & $38,8(40 / 103)$ \\
\hline RM prévia, \% (n/total) & $18,4(19 / 103)$ \\
\hline \multicolumn{2}{|l|}{ Apresentação clínica, \% (n/total) } \\
\hline Angina estável/isquemia silenciosa & $47,6(49 / 103)$ \\
\hline Síndrome coronariana aguda & $52,4(54 / 103)$ \\
\hline Angina instável & $23,3(24 / 103)$ \\
\hline IAM sem supra do segmento ST & $17,5(18 / 103)$ \\
\hline IAM com supra do segmento ST & $11,7(12 / 103)$ \\
\hline História familiar positiva para DAC, \% (n/total) & $33,0(34 / 103)$ \\
\hline \multicolumn{2}{|l|}{ Doença coronária, \% (n/total) } \\
\hline Uniarterial & $36,9(38 / 103)$ \\
\hline Biarterial & $33,0(34 / 103)$ \\
\hline Triarterial & $30,1(31 / 103)$ \\
\hline Número de lesões por paciente & $1,62 \pm 0,74$ \\
\hline \multicolumn{2}{|l|}{ Uso de aspirina, \% (n/total) } \\
\hline Intra-hospitalar & $56,3(58 / 103)$ \\
\hline 30 dias & $90,7(78 / 86)$ \\
\hline 6 meses & $90,4(75 / 83)$ \\
\hline 1 ano & $80,2(77 / 96)$ \\
\hline \multicolumn{2}{|l|}{ Uso de clopidogrel, \% (n/total) } \\
\hline Intra-hospitalar & $43,7(45 / 103)$ \\
\hline 30 dias & $95,3(82 / 86)$ \\
\hline 6 meses & $92,8(77 / 83)$ \\
\hline 1 ano & $79,2(76 / 96)$ \\
\hline
\end{tabular}

IAM : infarto agudo do miocárdio; ATC: angioplastia transluminal coronária; RM: revascularização do miocárdio; DAC: doença arterial coronária.

\section{Análise estatística}

$\mathrm{Na}$ análise estatística descritiva, as variáveis categóricas foram expressas como frequências absolutas e percentuais. As variáveis contínuas foram expressas como média \pm desvio-padrão. As análises de sobrevida foram representadas de acordo com as curvas de Kaplan-Meier e, para a comparação entre os grupos, utilizou-se o teste estatístico de log-rank. $\mathrm{O}$ valor de $\mathrm{p}$ foi considerado significativo quando $<0,05$. Para realizar tais análises, foi utilizado o pacote estatístico Statistical Package for the Social Science (SPSS) 13.0.

Tabela 2. Características angiográficas iniciais

\begin{tabular}{|c|c|}
\hline & $n=152$ \\
\hline \multicolumn{2}{|l|}{ Artéria tratada, \% (n/total) } \\
\hline Descendente anterior & $49,7(75 / 151)$ \\
\hline Coronária direita & $18,5(28 / 151)$ \\
\hline Circunflexa & $23,8(36 / 151)$ \\
\hline Ponte safena & $6,6(10 / 151)$ \\
\hline Ponte mamária & $0,7(1 / 151)$ \\
\hline Tronco da coronária não protegida & $0,7(1 / 151)$ \\
\hline \multicolumn{2}{|l|}{ Local da lesão, \% (n/total) } \\
\hline Ostial & $13,8(21 / 152)$ \\
\hline Proximal & $49,3(75 / 152)$ \\
\hline Média & $23,7(36 / 152)$ \\
\hline Distal & $13,2(20 / 152)$ \\
\hline \multicolumn{2}{|l|}{ Tipo da lesão, \% (n/total) } \\
\hline Estenose de novo & $87,3(131 / 150)$ \\
\hline Reestenose intra-stent & $12,7(19 / 150)$ \\
\hline \multicolumn{2}{|l|}{ Valores de referência iniciais do vaso-alvo } \\
\hline Diâmetro de referência do vaso, mm & $2,43 \pm 0,60(n=148)$ \\
\hline Diâmetro luminal mínimo, mm & $0,94 \pm 0,54(n=145)$ \\
\hline Diâmetro de estenose, \% & $62,41 \pm 20,41(n=148)$ \\
\hline Extensão da lesão, mm & $17,40 \pm 11,91(n=137)$ \\
\hline \multicolumn{2}{|l|}{ Características do vaso-alvo, \% (n/total) } \\
\hline Angulação > 45º & $6,6(10 / 152)$ \\
\hline Angulação 45-90 & $4,6(7 / 152)$ \\
\hline Angulação > 90 & $2,0(3 / 152)$ \\
\hline Trombo prévio & $5,9(9 / 152)$ \\
\hline Tortuosidade moderada/grave & $6,6(10 / 152)$ \\
\hline Calcificação moderada/grave & $23,7(36 / 152)$ \\
\hline Ulceração & $2,0(3 / 152)$ \\
\hline Flap intimal & $2,0(3 / 152)$ \\
\hline Ectasia & $1,3(2 / 152)$ \\
\hline Bifurcação & $16,4(25 / 152)$ \\
\hline \multicolumn{2}{|l|}{ TIMI prévio, \% (n/total) } \\
\hline $0 / 1$ & $7,9(12 / 152)$ \\
\hline 2 & $6,6(10 / 152)$ \\
\hline 3 & $84,2(128 / 152)$ \\
\hline
\end{tabular}

TIMI: thrombolysis in myocardial infarction. 


\section{RESULTADOS}

\section{Características clínicas e do procedimento}

As características clínicas iniciais são apresentadas na tabela 1. A média de idade da população foi de $65,0 \pm 12,4$ anos, com predomínio do gênero masculino $(83,5 \%)$. Dentre os fatores de risco para doença arterial coronária (DAC), ressaltam-se elevada prevalência de diabetes mellitus (37,9\%), hipertensão arterial sistêmica $(75,7 \%)$ e hipercolesterolemia $(70,9 \%)$. A apresentação clínica mais prevalente foi de angina estável/isquemia silenciosa $(47,6 \%)$, seguida de angina instável $(23,3 \%)$. Angioplastia primária no infarto agudo do miocárdio (IAM) foi realizada em 11,7\% dos pacientes.

Dados relativos ao procedimento terapêutico, assim como características angiográficas das lesões tratadas estão descritas nas tabelas 2 e 3 . A maioria dos pacientes tratados era multiarterial (63\%), incluindo um terço dos pacientes com DAC triarterial. O tratamento incluiu lesões de novo, reestenose intra-stent $(12,7 \%)$ e o tratamento de lesões de bifurcação $(16,4 \%)$.

Tabela 3. Características angiográficas pós-procedimento

\begin{tabular}{|c|c|}
\hline & $n=152$ \\
\hline \multicolumn{2}{|l|}{ Fatores relacionados ao procedimento } \\
\hline Diâmetro do stent, mm & $3,09 \pm 0,43(n=148)$ \\
\hline Comprimento total do stent, mm & $23,19 \pm 11,97(n=148)$ \\
\hline $\begin{array}{l}\text { Razão de comprimento do stent por } \\
\text { comprimento da lesão, } \mathrm{mm} / \mathrm{mm}\end{array}$ & $1,57 \pm 0,76(n=119)$ \\
\hline Número de stents por lesão & $1,16 \pm 0,50(n=152)$ \\
\hline \multicolumn{2}{|l|}{ Valores de referência finais do vaso-alvo } \\
\hline Diâmetro de referência do vaso, mm & $2,91 \pm 0,61(n=148)$ \\
\hline Diâmetro luminal mínimo, mm & $2,23 \pm 0,58(n=148)$ \\
\hline Diâmetro da estenose, \% & $23,41 \pm 9,77(n=148)$ \\
\hline Diâmetro luminal mínimo intra-stent, mm & $2,48 \pm 0,53(n=148)$ \\
\hline Diâmetro de estenose intra-stent, \% & $15,57 \pm 7,21(n=148)$ \\
\hline \multicolumn{2}{|l|}{ TIMl final, \% (n/total) } \\
\hline $0 / 1$ & $1,3(2 / 152)$ \\
\hline 2 & $0,7(1 / 152)$ \\
\hline 3 & $98,0(149 / 152)$ \\
\hline \multicolumn{2}{|l|}{ Achados finais da lesão alvo, \% (n/total) } \\
\hline Trombo & $0,0(0 / 152)$ \\
\hline Embolização distal & $0,7(1 / 152)$ \\
\hline Oclusão aguda & $0,0(0 / 152)$ \\
\hline No reflow & $0,0(0 / 152)$ \\
\hline Perfuração & $0,7(1 / 152)$ \\
\hline Dissecção & $0,7(1 / 152)$ \\
\hline
\end{tabular}

TIMI: thrombolysis in myocardial infarction.

\section{Seguimento clínico}

Dados relativos ao seguimento intra-hospitalar, de 30 dias e 12 meses após o procedimento índice foram obtidos de $98 \%$ dos pacientes por meio de consulta ambulatorial ou contato telefônico. Na tabela 4, estão descritos os achados para cada período do primeiro ano de seguimento.

Tabela 4. Seguimento intra-hospitalar, 30 dias e 12 meses e os respectivos eventos cumulativos

\begin{tabular}{|c|c|}
\hline & $\%$ (n/total) \\
\hline \multicolumn{2}{|l|}{ Intra-hospitalar } \\
\hline EACM & $4,9(5 / 103)$ \\
\hline Óbito & $1,0(1 / 103)$ \\
\hline Cardíaco & $1,0(1 / 103)$ \\
\hline Não cardíaco & $0,0(0 / 103)$ \\
\hline IAM & $3,9(4 / 103)$ \\
\hline Sem supra do segmento ST & $3,9(4 / 103)$ \\
\hline Com supra do segmento ST & $0,0(0 / 103)$ \\
\hline RM & $0,0(0 / 103)$ \\
\hline Do vaso-alvo & $0,0(0 / 103)$ \\
\hline Do vaso não alvo & $0,0(0 / 103)$ \\
\hline Trombose de stent & $1,0(1 / 103)$ \\
\hline AVC & $0,0(0 / 103)$ \\
\hline Complicações vasculares & $0,0(0 / 105)$ \\
\hline \multicolumn{2}{|l|}{30 dias } \\
\hline EACM & $5,8(6 / 103)$ \\
\hline Óbito & $1,9(2 / 103)$ \\
\hline Cardíaco & $1,9(2 / 103)$ \\
\hline Não cardíaco & $0,0(0 / 103)$ \\
\hline IAM & $3,9(4 / 103)$ \\
\hline RM & $1,0(1 / 103)$ \\
\hline Do vaso-alvo & $0,0(0 / 103)$ \\
\hline Da lesão alvo & $0,0(0 / 103)$ \\
\hline Do vaso não alvo & $1,0(1 / 103)$ \\
\hline Trombose de stent & $1,0(1 / 103)$ \\
\hline AVC & $0,0(0 / 103)$ \\
\hline \multicolumn{2}{|l|}{12 meses } \\
\hline EACM & $11,7(12 / 103)$ \\
\hline Óbito & $5,8(6 / 103)$ \\
\hline Cardíaco & $2,9(3 / 105)$ \\
\hline Não cardíaco & $2,9(3 / 105)$ \\
\hline IAM & $4,9(5 / 103)$ \\
\hline RM & $6,8(7 / 103)$ \\
\hline Do vaso-alvo & $3,9(4 / 103)$ \\
\hline Da lesão alvo & $3,9(4 / 103)$ \\
\hline Do vaso não alvo & $2,9(3 / 103)$ \\
\hline Trombose de stent & $1,0(1 / 103)$ \\
\hline AVC & $1,0(1 / 103)$ \\
\hline
\end{tabular}


Após 12 meses de seguimento clínico, foram detectados eventos adversos cardíacos maiores (EACM) em 12 pacientes, 6 óbitos ( 3 de origem cardíaca e 3 de origem não cardíaca), 5 casos de IAM sem supradesnivelamento do segmento ST e nova revascularização do vaso-alvo (RVA) em quatro pacientes. Entretanto, é fundamental destacar a baixa incidência de trombose de stent, tendo sido diagnosticado apenas um caso após o primeiro ano do implante do stent. Esse único caso ocorreu nas primeiras 24 horas após a angioplastia (trombose aguda), não tendo ocorrido nenhum caso de trombose tardia e/ou muito tardia.

Na figura 1, observa-se a curva de Kaplan-Meyer demonstrando a sobrevida livre de eventos em todos os

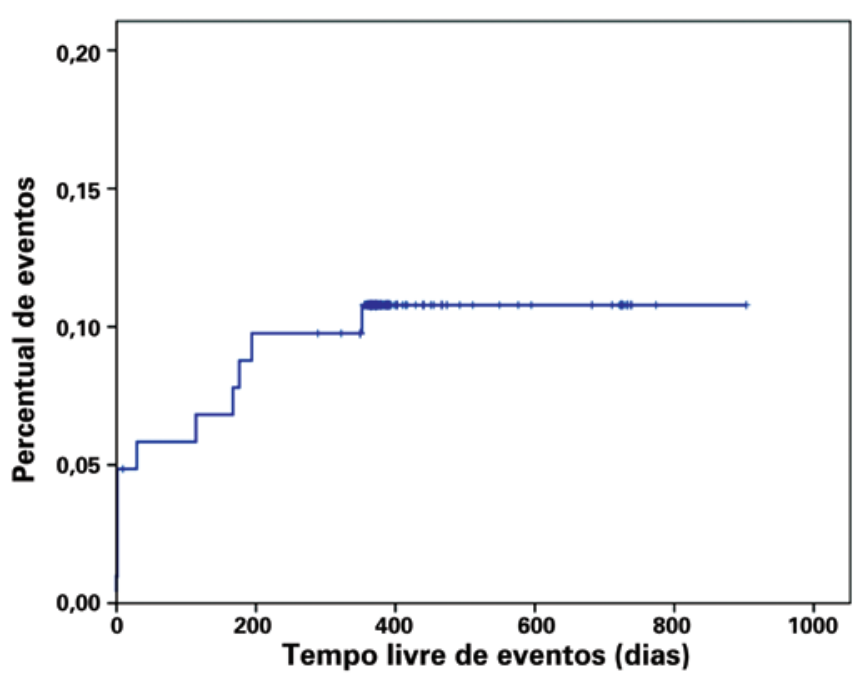

Figura 1. Curva de Kaplan-Meier demonstrando a taxa de eventos em todos os pacientes

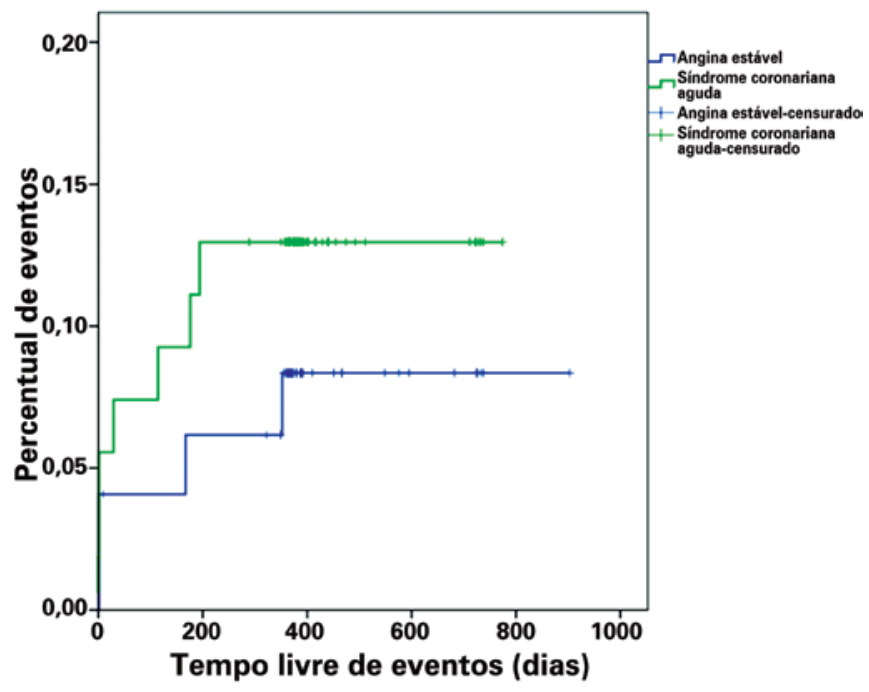

Figura 2. Curva de Kaplan-Meier demonstrando a taxa de eventos no grupo de pacientes com angina estável e síndrome coronária aguda pacientes do estudo. Cabe ressaltar a diferença encontrada, ainda que sem significância estatística, quanto à sobrevida livre de eventos entre os grupos de apresentação síndrome coronária aguda e angina estável $(87,0 \%$ versus $91,8 \% ; \mathrm{p}=0,43$ ) (Figura 2). Com relação ao padrão de distribuição da doença coronária (uniarterial ou multiarterial), ressalta-se o padrão similar, não havendo diferença em termos de sobrevida livre de eventos entre os dois grupos $(89,5 \%$ versus $89,2 \% ; p=0,97)$ (Figura 3).

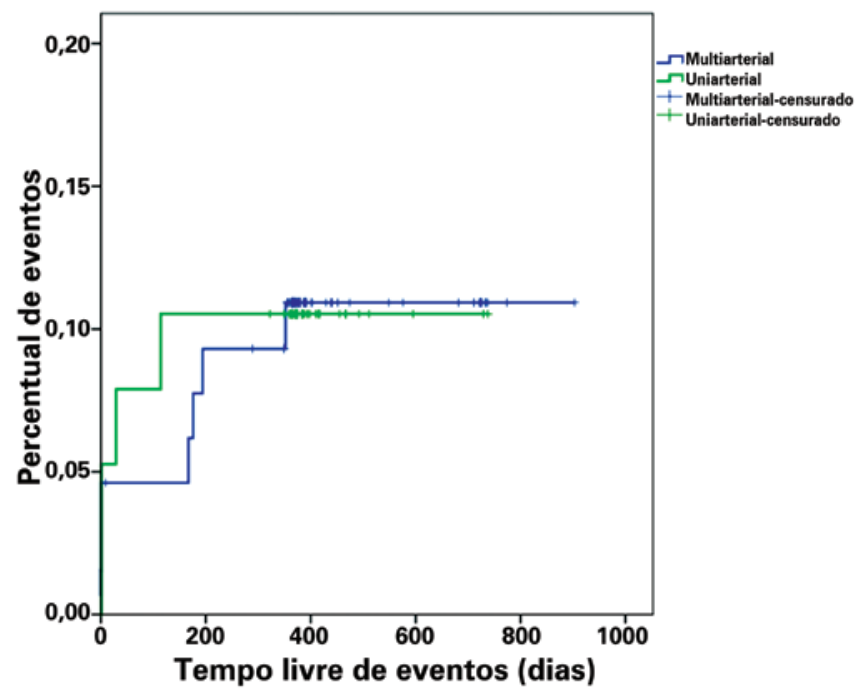

Figura 3. Curva de Kaplan-Meier demonstrando a taxa de eventos no grupo uniarterial e multiarterial

\section{DISCUSSÃO}

Os resultados do presente manuscrito corroboram e adicionam novos dados àqueles já publicados anteriormente sobre a utilização do stent eluidor de biolimus A9

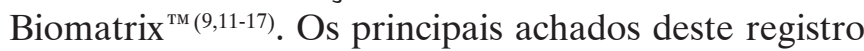
são: em uma população de pacientes consecutivos do "mundo real", incluindo pacientes de alta complexidade clínica (diabéticos) e anatômica (multiartérias, bifurcações, lesões reestenóticas e IAM com supradesnivelamento do segmento ST), o stent eluidor de biolums A9 apresenta-se seguro e eficaz no seguimento de longo prazo.

A presença de um polímero durável e pouco biocompatível nos SFs de primeira geração esteve associado à inflamação e à reação de hipersensibilidade vascular, ao comprometimento do processo de re-endotelização e, importantemente, à trombose tardia e morte. O biolimus é um análogo sintético do sirolimus, com uma lipofilicidade dez vezes superior a este e com similar potência antiproliferativa. O polímero de ácido 
polilático é aplicado apenas na superfície abluminal e, após a total liberação do fármaco, é completamente dissolvido em gás carbônico e água em um período de 6 a 9 meses. Essas vantagens farmacodinâmicas e de bioengenharia o tornam, em tese, uma plataforma mais segura que os polímeros duráveis utilizados nos SFs convencionais. O estudo de fase II STEALTH ${ }^{(11)}$ conduzido por Grube et al. foi o primeiro trabalho a avaliar a segurança e a eficácia do stent Biomatrix ${ }^{\mathrm{TM}}$. De forma randomizada e duplo-cega, o estudo incluiu 120 pacientes e chegou a resultados extremamente favoráveis ao grupo Biomatrix ${ }^{\mathrm{TM}}$ (perda luminal tardia intra-stent de $0,26 \mathrm{~mm}$ ) quando comparado ao grupo controle $(0,74 \mathrm{~mm})$. A sobrevida livre de eventos aos 6 meses foi similar entre os dois grupos. Esses primeiros resultados impulsionaram o desenvolvimento do programa clínico LEADERS $^{(9)}$, que randomizou, de maneira prospectiva, 1.707 pacientes em dez centros na Europa. Nesse estudo, a segurança e a eficácia do stent Biomatrix ${ }^{\mathrm{TM}}$ (grupo BES) foram comparadas as de um stent de primeira geração eluidor de sirolimus com polímero durável (Cypher SELECT ${ }^{\mathrm{TM}}$, Cordis, Miami Lakes, FL, USA). Aos 9 meses, as taxas de EACM (morte cardíaca, IAM ou RVA) no grupo BES foi estatisticamente não inferior às do stent eluidor de sirolimus (SES). Além disso, o BES foi não inferior ao SES em relação ao percentual de diâmetro de estenose intra-stent $(20,9 \%$ versus $23,3 \%$ ) no mesmo período. Neste estudo, é importante ressaltar as taxas similares de trombose definitiva de stent tanto aguda e subaguda (1,6\% para o BES versus $1,6 \%$ para o SES) quanto tardia e muito tardia (0,2\% para o BES versus $0,5 \%$ para o SES). A presente análise demonstra taxas de eventos isquêmicos similares aos resultados do estudo LEADERS, a despeito da maior inclusão de pacientes diabéticos ( $1 / 3$ versus $25 \%$ de diabéticos no estudo LEADERS), presença de IAM com supradesnivelamento de segmento ST e vasos de fino calibre (2,4mm versus $2,6 \mathrm{~mm}$ no LEADERS). De nota, observa-se apenas um caso de trombose subaguda do stent (relacionada ao procedimento) e nenhum caso de trombose tardia e/ou muito tardia. Assim, apesar do pequeno número de paciente incluídos neste registro, o presente estudo sugere que essa plataforma de stent com polímero biodegradável é segura em pacientes de alto risco no longo prazo.

Entretanto, como o risco de trombose de SF de primeira geração sofre possivelmente um incremento a partir do primeiro ano do implante, dados de seguimento mais longos seriam necessários para confirmar a não inferioridade e/ou superioridade do polímero biodegradável. Dessa forma, com o seguimento de 4 anos do estudo LEADERS ${ }^{(12)}$, observou-se, mais uma vez, a não inferioridade do stent eluidor de biolimus em relação ao desfecho primário para o BES $(18,7 \%)$ versus o SES $(22,6 \%)$. Adicionalmente, houve uma redução de $38 \%$ no risco relativo de trombose de stent definitiva entre 1 e 4 anos no grupo BES quando comparado ao grupo SES. A maior segurança da plataforma Biomatrix ${ }^{\mathrm{TM}}$ utilizando o polímero biodegradável, quando comparada aos SF convencionais com polímero durável, pode estar relacionada a uma mais completa cobertura das hastes dos stents no longo prazo, como demonstrado em estudo com tomografia de coerência óptica ${ }^{(13)}$.

Importantemente, o stent Biomatrix ${ }^{\mathrm{TM}}$ também teve seu desempenho e sua segurança avaliados em subtipos específicos de pacientes. Quando os pacientes foram divididos de acordo com o Syntax Score (SX) em 3 grupos ( $\mathrm{SX}$ baixo se $\leq 8$, SX médio se $>8$ e $\leq 16$ e $\mathrm{SX}$ alto se $>16$ ), observaram-se as seguintes taxas de EACM após 2 anos de seguimento: 9,4\% para SX baixo, $12,0 \%$ para SX médio e $18,4 \%$ para SX alto $(\mathrm{p}<0,01)$. Apesar da taxa de óbito cardíaco nos pacientes com SX alto ser significativamente maior quando comparada aos outros dois grupos ( $7 \%$ versus $2,4 \%$ versus $1,8 \%$ ), foi possível evidenciar que, nesse subgrupo de pacientes de maior complexidade anatômica, aqueles tratados com BES apresentaram taxas significativamente menores de óbito cardíaco quando comparados ao grupo tratado com SES ${ }^{(14)}$. Por outro lado, na análise de pacientes com vasos de pequeno calibre (diâmetro do vaso $<2,75 \mathrm{~mm}$ ), não houve diferença significativa nas taxas de EACM e nas taxas de RVA quando comparados os dois grupos $^{(15)}$. Finalmente, na análise de subgrupo de pacientes com IAM com supradesnivelamento do segmento ST, houve redução significativa de EACM nos pacientes tratados com BES comparado àqueles com $\operatorname{SES}(8,1 \%$ versus $19,3 \%)^{(16)}$

Soares Junior et al. ${ }^{(17)}$ publicaram resultados similares aos encontrados na presente análise após seguimento clínico médio de 243 dias em 100 pacientes (164 lesões) tratados com stent Biomatrix ${ }^{\mathrm{TM}}$. Nesse grupo de pacientes, o desfecho primário ocorreu em $9 \%$ dos pacientes (óbito cardíaco em $4 \%$, IAM não fatal em $2 \%$ e RVA em 3\%) e a taxa de trombose de stent encontrada foi de $1 \%$. Dessa maneira, como o presente estudo, não foram observadas tromboses tardias ou muito tardias nessa coorte de pacientes.

Recentemente, o estudo COMPARE II ${ }^{(18)}$ evidenciou a segurança e a eficácia dos SF eluidores de bilomus com polímero biodegradável Nobori ${ }^{\mathrm{TM}}$ (Terumo Corporation, Tokyo, Japan) comparado aos SF convencionais de nova geração utilizando o everolimus e o polímero durável (Xience $\mathrm{V}^{\mathrm{TM}}$, Abbot Vascular, Santa Clara, CA, USA). Os resultados de segurança positivos 
do stent eluidor de biolimus deste estudo abrem uma nova perspectiva para a estratégia de dupla antiagregação plaquetária <1 ano, mesmo envolvendo pacientes de mais alto risco.

O presente estudo apresenta as seguintes limitações. Trata-se de estudo observacional, realizado em um único centro e sem um controle ativo comparativo. $\mathrm{O}$ número de pacientes incluídos foi pequeno e as conclusões são apenas exploratórias, necessitando estudos comprobatórios futuros. A inclusão de pacientes ficou a critério dos operadores, por isso não foi possível afastar a possibilidade de viés de seleção na escolha do stent no momento do procedimento. Finalmente, o tempo de seguimento clínico ainda foi curto; a minoria dos paciente possuía seguimento superior a 1 ano. Portanto, os dados de segurança e eficácia não podem ser extrapolados aos de um acompanhamento de maior longo prazo.

\section{CONCLUSÕES}

Os novos stents farmacológicos eluidores de biolimus A9 com polímero biodegradável são seguros e eficazes em pacientes da prática clínica diária do "mundo real", incluindo aqueles com alta complexidade clínica e anatômica, apresentando baixas taxas de eventos isquêmicos no longo prazo.

\section{AGRADECIMENTO}

Estudo financiado parcialmente pela Bioassist Medical LTDA, para análise angiográfica quantitativa.

\section{REFERÊNCIAS}

1. Schwietz T, Ehrlich JR, De Rosa S, Fichtlscherer S, Schachinger V, Baier $G$, et al. Prognostic impact of using drug-eluting-stents on outcome and strategy in multivessel PCI: Data from the Frankfurt MV-PCI registry. J Cardiol. 2013;61(1):38-43.

2. Bangalore S, Kumar S, Fusaro M, Amoroso N, Attubato MJ, Feit F, et al. Shortand long-term outcomes with drug-eluting and bare-metal coronary stents: a mixed-treatment comparison analysis of 117762 patient-years of follow-up from randomized trials. Circulation. 2012;125(23):2873-91.

3. Yeh RW, Normand SL, Wolf RE, Jones PG, Ho KK, Cohen DJ, et al. Predicting the restenosis benefit of drug-eluting versus bare metal stents in percutaneous coronary intervention. Circulation. 2011;124(14):1557-64.

4. Camenzind E, Wijns W, Mauri L, Kurowski V, Parikh K, Gao JP, Boersma E, Vranckx P, McFadden E, Serruys PW, O'Neil WW, Jorissen B, Van Leeuwen F, Steg PG; PROTECT Steering Committee and Investigators.
Stent thrombosis and major clinical events at 3 years after zotarolimuseluting or sirolimus-eluting coronary stent implantation: a randomised, multicentre, open-label, controlled trial. Lancet. 2012;380(9851):1396-405.

5. Karjalainen PP, Nammas W, Ylitalo A. Late stent thrombosis of a secondgeneration drug-eluting stent. J Invasive Cardiol. 2012;24(10):E225-7.

6. Räber L, Magro M, Stefanini GG, Kalesan B, van Domburg RT, Onuma Y, et al. Very late coronary stent thrombosis of a newer-generation everolimuseluting stent compared with early-generation drug-eluting stents: a prospective cohort study. Circulation. 2012;125(9):1110-21.

7. Chen JP, Hou D, Pendyala L, Goudevenos JA, Kounis NG. Drug-eluting stent thrombosis: the Kounis hypersensitivity-associated acute coronary syndrome revisited. JACC Cardiovasc Interv. 2009;2(7):583-93.

8. Siddiqi OK, Faxon DP. Very late stent thrombosis: current concepts. Curr Opin Cardiol. 2012;27(6):634-41.

9. Windecker S, Serruys PW, Wandel S, Buszman P, Trznadel S, Linke A, et al Biolimus-eluting stent with biodegradable polymer versus sirolimus-eluting stent with durable polymer for coronary revascularisation (LEADERS): a randomized non-inferiority trial. Lancet. 2008;372(9644):1163-73.

10. Cutlip DE, Windecker S, Mehran R, Boam A, Cohen DJ, van Es GA, et al Academy Research Consortium. Clinical end points in coronary stent trials. A case for standardized definitions. Circulation. 2007;115(17):2344-51.

11. Grube E, Hauptmann KE, Buellesfeld L, Lim V, Abizaid A. Six-month results of a randomized study to evaluate safety and efficacy of a Biolimus A9 eluting stent with a biodegradable polymer coating. Eurolntervention. 2005;1 (1):53-7.

12. Stefanini GG, Kalesan B, Serruys PW, Heg D, Buszman P, Linke A, et al. Long-term clinical outcomes of biodegradable polymer biolimus-eluting stents versus durable polymer sirolimus-eluting stents in patients with coronary artery disease (LEADERS): 4 year follow-up of a randomised noninferiority trial. Lancet. 2011;378(9807):1940-8.

13. Barlis P, Regar E, Serruys PW, Dimopoulos K, van der Giessen WJ, van Geuns RJ, et al. An optical coherence tomography study of a biodegradable vs. durable polymer-coated limus-eluting stent: a LEADERS trial sub-study. Eur Heart J. 2010;31(2):165-76.

14. Wykrzykowska JJ, Garg S, Onuma Y, de Vries T, Morel MA, van Es GA, et al. Implantation of the biodegradable polymer biolimus-eluting stent in patients with high SYNTAX score is associated with decreased cardiac mortality compared to a permanent polymer sirolimus-eluting stent: two year follow-up results from the "all-comers" LEADERS trial. Eurolntervention. 2011;7(5):605-13.

15. Wykrzykowska JJ, Serruys PW, Onuma Y, de Vries T, van Es GA, Buszman $P$, et al. Impact of vessel size on angiographic and clinical outcomes of revascularization with biolimus-eluting stent with biodegradable polymer and sirolimus-eluting stent with durable polymer the LEADERS trial substudy. JACC CardiovascInterv. 2009;2(9):861-70.

16. Raber L, Kelbaek H, Ostojic M, Baumbach A, Heg D, Tuller D, et al. Effect of biolimus-eluting stents with biodegradable polymer vs bare-metal stents on cardiovascular events among patients with acute myocardial infarction: the COMFORTABLE AMI randomized trial. JAMA. 2012;308(8)777-87.

17. Soares Junior RS, Majeski JD, Carnieto NM, Zuliani MF, Lapa GA, Bavaresco $S A$, et al. Resultados da intervenção coronária percutânea com 0 stent

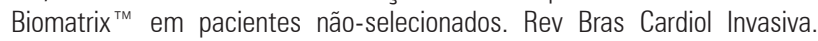
2011;19(1):40-6.

18. Smits P, van Boven AJ, Valdes M, Serra A, Goy JJ, Voudris V, et al. TCT573 Randomized comparison of Biolimus-eluting (Nobori) and Everolimuseluting (Xience/Promus) stents in patients with multivessel coronary artery disease: 12-month follow-up data from COMPARE II study. J Am Coll Cardiol. 2012;60(17 S). 